\title{
Solution of the generalised random energy model
}

\author{
$B$ Derrida $\uparrow$ and E Gardner $\pitchfork$ \\ $\uparrow$ SPT, CEN Saclay, 91191 Gif sur Yvette, France \\ $\doteqdot$ Department of Physics, Edinburgh University, Edinburgh EH9 3JZ, UK
}

Received 30 August 1985, in final form 16 October 1985

\begin{abstract}
The generalised random energy model (GREM) is a spin-glass model which can be solved exactly. One can impose arbitrary pair correlations between the energies of configurations. For several examples (the Sherrington-Kirkpatrick model, the p spin-glass model, the Potts glass, spin-glass models on finite-dimensional lattices) we calculate the pair correlations between energies and solve the corresponding GREM. In all cases, the free energy of the GREM corresponding to a spin-glass model on a given lattice, has a simple expression in terms of the specific heat of the pure ferromagnetic model on the same lattice. Lastly we compare the correlations between three energy levels in the GREM and in spin-glass models.
\end{abstract}

\section{Introduction}

In the last ten years, the mean-field theory of spin glasses has motivated a lot of work (Proc. Heidelberg Conference 1983, Chowdhury and Mookerjee 1984). The Sherrington-Kirkpatrick (SK) model (Sherrington and Kirkpatrick 1975, 1978) has been most studied and numerous approaches have been developed in order to solve it. Although a full analytic solution of the SK model does not exist at present, the most promising approach seems to be the one based on the idea of a broken symmetry in replica space (Parisi 1980a, b, c, Blandin et al 1980). There does not yet exist a mathematical theory which justifies these replica calculations. However, the Parisi approach led to important ideas in the mean-field theory of spin glasses: ultrametricity (Mezard et al 1984) nonself-averaging effects (Mezard et al 1985), the probability distribution of overlaps (Parisi 1983). Thus, it is important to know whether one can exhibit a model which would possess all of these features but which could be solved exactly without using replicas.

The random energy model (REM), which is a spin-glass model simpler than the SK model, was introduced a few years ago (Derrida 1980). The REM is simple enough to be solved exactly without recourse to replicas. It nevertheless possesses some important effects which are observed in real spin glasses: a freezing temperature, a cusp in the magnetic susceptibility at the transition, a constant susceptibility in the spin-glass phase, the persistence of a transition in the presence of a magnetic field (Derrida 1981). Moreover, it was shown recently that features like ultrametricity (Gross and Mezard 1984) or non-self-averaging effects were also present in the REM (Derrida and Toulouse 1985) at least in embryo.

The REM consists in considering that the energies of the spin configuration are independent random variables. This is a very drastic simplification since one knows that 
in more realistic spin-glass models, these energies are strongly correlated (Derrida 1981). Thus it was interesting to see whether one could generalise the REM in order to study the effect of these correlations. It was shown recently that a generalised random energy model GREM (Derrida 1985) can be defined which possesses arbitrary pair correlations between the energies of the spin configurations.

The purpose of this paper is first to give a general solution of the GREM. Then we shall consider several examples of spin-glass models (the SK model with Ising spins, with Potts spins, the $\mathrm{p}$ spin-glass models, spin-glass models on finite-dimensional lattices) and for each case we shall solve the GREM which possesses the same pair correlations between the energies of configurations. Lastly we shall compare the three energy correlations of some of these spin-glass models with those of the corresponding GREM.

\section{Definition of the generalised random energy model}

Let us recall in this section the definition of the GREM. The GREM can be defined in the following way: one considers a system which consists of $2^{N}$ configurations: configuration $\nu$ has an energy $E_{\nu}\left(1 \leqslant \nu \leqslant 2^{N}\right)$. These configurations are grouped according to a hierarchy of $n$ levels. The $2^{N}$ configurations are grouped into groups of $\left(\alpha_{n}\right)^{N}$ configurations each: there are of course $\left(2 / \alpha_{n}\right)^{N}$ such groups at the $n$th level of the hierarchy. At the next level (level $n-1$ ), one regroups these groups: each group at the $(n-1)$ st level contains $\left(\alpha_{n-1}\right)^{N}$ groups at level $n$. Therefore at level $n-1$ of the hierarchy, there are $\left[2 /\left(\alpha_{n-1} \alpha_{n}\right)\right]^{N}$ groups of $\left(\alpha_{n-1} \alpha_{n}\right)^{N}$ configurations each. One can repeat this grouping procedure. At the $i$ th level, there are $\left[2 /\left(\alpha_{i} \alpha_{i+1} \ldots \alpha_{n}\right)\right]^{N}$ groups of $\left(\alpha_{i} \alpha_{i+1} \ldots \alpha_{n}\right)^{N}$ configurations each. Since altogether there are $2^{N}$ levels, one must have,

$$
\log \alpha_{1}+\log \alpha_{2}+\ldots+\log \alpha_{n}=\log 2 .
$$

The numbers $\alpha_{1}, \alpha_{2} \ldots \alpha_{n}$ are a priori arbitrary numbers larger than one. In the thermodynamic limit ( $N$ large) $\alpha_{1}^{N}, \alpha_{2}^{N} \ldots \alpha_{n}^{N}$ are large and so can be taken as integers.

One can see that the hierarchy constructed in this way has an obvious ultrametric structure (Mezard et al 1984). One can choose a priori an arbitrary sequence $q_{i}$ of overlaps between configurations

$$
0=q_{1}<q_{2}<q_{3}<\ldots<q_{i}<\ldots<q_{n+1}=1 .
$$

The overlap $q^{\mu \nu}$ between two configurations $\mu$ and $\nu$ is by definition equal to $q_{i}$ if the two configurations $\mu$ and $\nu$ belong to the same group at the $i$ th level of the hierarchy and belong to different groups at level $i+1$. The minimal overlap between configurations is $q_{1}$ and therefore we choose arbitrarily $q_{1}=0$. The maximal overlap $q_{n+1}$ is the overlap of a configuration with itself and therefore we choose $q_{n+1}=1$. The actual values of the $q_{i}$ do not matter much (since one can always define different metrics which are equivalent). The only important aspect is that the sequence $q_{i}$ is an increasing function of $i$ because this expresses the fact that the closer two configurations are, the larger their overlap is.

Let us now say how the values of the $2^{N}$ energies $E_{\nu}$ are chosen. By definition of the model, $E_{\nu}$ is the sum of $n$ random numbers $\varepsilon_{l}^{(\nu)}$

$$
E_{\nu}=\varepsilon_{1}^{(\nu)}+\varepsilon_{2}^{(\nu)}+\ldots+\varepsilon_{n}^{(\nu)}
$$

where $\varepsilon_{l}^{(\nu)}$ is a random number distributed according to a given probability distribution $\rho_{i}$. 


$$
\rho_{i}\left(\varepsilon_{i}\right)=\left(\pi N J^{2} a_{i}\right)^{-1 / 2} \exp \left(-\varepsilon_{i}^{2} / N J^{2} a_{i}\right)
$$

$\varepsilon_{i}^{(\nu)}$ is the contribution to $E_{\nu}$ coming from the $i$ th level of the hierarchy. Note that the width $a_{i}$ of the distribution $\rho_{i}$ depends on $i$. By defintion of the model, two energies $E_{\mu}$ and $E_{\nu}$ which belong to the same group at the $i$ th level of the hierarchy have the same $\varepsilon$ , for $j \leqslant i-1$ but have different $\varepsilon_{j}$ for $j \geqslant i$ if they belong to different groups at the $(i+1)$ st level

$$
\begin{array}{ll}
\varepsilon_{j}^{(\nu)}=\varepsilon_{j}^{(\mu)} & \text { for } j \leqslant i-1 \\
\varepsilon_{j}^{(\nu)} \neq \varepsilon_{j}^{(\mu)} & \text { for } j \geqslant i .
\end{array}
$$

Another way of saying this is to say that equation (5) holds when

$$
q^{\mu \nu}=q_{i}
$$

So two configurations which have all their $\varepsilon_{i}$ different have an overlap $q_{1}=0$. On the other hand two configurations which have all the $\varepsilon_{i}$ equal are in fact the same configuration and have an overlap $q_{n+1}=1$. When the $\varepsilon_{j}^{(\nu)}$ and $\varepsilon_{j}^{(\mu)}$ are different, they are independent. Also $\varepsilon_{j}^{(\nu)}$ and $\varepsilon_{k}^{(\mu)}$ are always independent for $j \neq k$.

The model is defined once the two sequences $\alpha_{i}$ and $a_{i}$ are given for $1 \leqslant i \leqslant n$. To normalise the energies, we shall impose the condition

$$
\sum_{i=1}^{n} a_{i}=1
$$

We can now calculate the probability distribution of energy levels. Since $E_{v}$ is a sum of independent gaussian variables (see equations (3), (4) and (7)) the probability distribution $P_{\nu}\left(E_{\nu}\right)$ is just

$$
P_{\nu}\left(E_{\nu}\right)=\left(\pi N J^{2}\right)^{-1 / 2} \exp -\left(E_{\nu}^{2} / N J^{2}\right) .
$$

One can also calculate the probability distribution $P_{\mu, \nu}\left(E_{\mu}, E_{\nu}\right)$ that two configurations $\mu$ and $\nu$ have energies $E_{\mu}$ and $E_{\nu}$. This probability distribution depends on the distance between configurations $\mu$ and $\nu$ in the hierarchy. One finds (Derrida 1985)

$$
P_{\mu, \nu}\left(E_{\mu}, E_{\nu}\right)=\text { const. } \exp \left(-\frac{\left(E_{\mu}+E_{\nu}\right)^{2}}{2 N(1+v) J^{2}}-\frac{\left(E_{\mu}-E_{\nu}\right)^{2}}{2 N(1-v) J^{2}}\right)
$$

where $v$ is given by

$$
v=\sum_{j=1}^{i-1} a_{j}
$$

and $i$ is the highest level in the hierarchy for which configurations $\mu$ and $\nu$ belong to the same group (i.e. $q^{\mu \nu}=q_{i}$ ). So $v$ is a measure of the correlation between two configurations with overlap $q_{i}$.

Given a configuration $\nu$, the number $\mathrm{e}^{N u}$ of configurations $\mu$ to which formulas (9) and (10) apply is just the number of configurations $\mu$ which have an overlap $q^{\mu \nu}=q_{i}$ with configuration $\nu$

$$
\mathrm{e}^{N u}=\left[\left(\alpha_{i}\right)^{N}-1\right]\left(\alpha_{i+1} \ldots \alpha_{n}\right)^{N} .
$$

In the thermodynamic limit, $(N \rightarrow \infty)$ one has

$$
u=\sum_{j=i}^{n} \log \alpha_{j}
$$


So we can see that by choosing the $\alpha_{i}$ and $a_{i}$ properly one can produce any probability distribution $P_{\mu, \nu}$. In the thermodynamic limit $(N \rightarrow \infty)$, one can have a very large number $n$ of levels in the hierarchy. One quantity of interest in the GREM is the function $g(y)$ defined by (Parisi 1983, Mezard et al 1984)

$g(y)=\overline{\left[\sum_{\mu, \nu} \exp \left(-\beta E_{\mu}-\beta E_{\nu}+y q^{\mu \nu}\right)\right] /\left[\sum_{\mu, \nu} \exp \left(-\beta E_{\mu}-\beta E_{\nu}\right)\right]}$.

$g(y)$ is the characteristic function of the distribution of overlaps $P(q)$. (The bars in equation (13) denote the average over disorder)

$$
g(y)=\int \mathrm{d} q \overline{P(q)} \mathrm{e}^{q y} .
$$

We are not yet able to calculate $g(y)$ for the GREM. Therefore in this paper we shall just say what we expect for its shape or more precisely for the shape of $q(x)$ which is related as usual to $\overline{P(q)}$ by

$$
1-x=\int_{q(x)}^{1} \mathrm{~d} q^{\prime} \overline{P\left(q^{\prime}\right)}
$$

\section{Solution of the generalised random energy model}

For $n=1$, the GREM reduces to the random energy model which can be solved exactly. For $n=2$, the GREM has been solved (Derrida 1985) and one finds that it exhibits two phase transitions if $\left(a_{1} / \log \alpha_{1}\right)>\left(a_{2} / \log \alpha_{2}\right)$ and one phase transition if $\left(a_{1} / \log \alpha_{1}\right)<\left(a_{2} / \log \alpha_{2}\right)$.

One can easily generalise that solution for arbitrary $n, \alpha_{i}$ and $a_{i}$. The solution is described in the appendix. Let us just give here the result in two cases which will be of interest later.

First, if the sequence $a_{i} / \log \alpha_{i}$ is decreasing

$$
\frac{a_{1}}{\log \alpha_{1}}>\frac{a_{2}}{\log \alpha_{2}}>\ldots>\frac{a_{n}}{\log \alpha_{n}}
$$

and if we define the temperatures $T_{i}$ by

$$
T_{i}=\frac{J}{2}\left(\frac{a_{i}}{\log \alpha_{i}}\right)^{1 / 2}
$$

then the free energy in the thermodynamic limit $(N \rightarrow \infty)$ is given by: if $T>T_{1}$

$$
\frac{1}{N} \overline{\log Z}=\log 2+J^{2} / 4 T^{2}
$$

if $T_{i}>T>T_{i+1}$

$$
\frac{1}{N} \overline{\log Z}=\sum_{j=i+1}^{n}\left(\log \alpha_{j}+a_{j} J^{2} / 4 T^{2}\right)+J / T \sum_{j=1}^{i}\left(a_{j} \log \alpha_{j}\right)^{1 / 2}
$$


if $T_{\mathrm{n}}>T$

$$
\frac{1}{N} \overline{\log Z}=(J / T) \sum_{j=1}^{n}\left(a_{j} \log \alpha_{j}\right)^{1 / 2} .
$$

For $T_{i}>T>T_{i+1}$, the system is frozen in a few groups at level $i+1$ but in each of these groups, it behaves as a system in its high temperature phase. In other words, in that range of temperature, the only configurations $\nu$ which contribute to the partition function are those for which the $\varepsilon_{j}^{v}$ (for $j \leqslant i$ ) are at a finite distance from their minimal possible value

$$
-\frac{\varepsilon_{j}^{(\nu)}}{N J}-\left(a_{j} \log \alpha_{j}\right)^{1 / 2}+\frac{1}{4}\left(\frac{a_{j}}{\log \alpha_{j}}\right)^{1 / 2} \frac{\log N}{N} \simeq 0\left(\frac{1}{N}\right) .
$$

This means that one has a finite probability that $q^{\mu \nu}$ takes the value $q_{j}$ for $j \leqslant i+1$. Therefore one expects that $q(x)$ will have the shape indicated in figure 1(a). When one decreases the temperature one expects that the discontinuities will move and that when $T$ crosses $T_{i+1}$, the value $q_{i+1}$ will appear in $q(x)$. We have not yet succeeded in calculating the location of the discontinuities of $q(x)$ which have more physical meaning than the $q_{i}$ (in the way they are defined in the GREM).
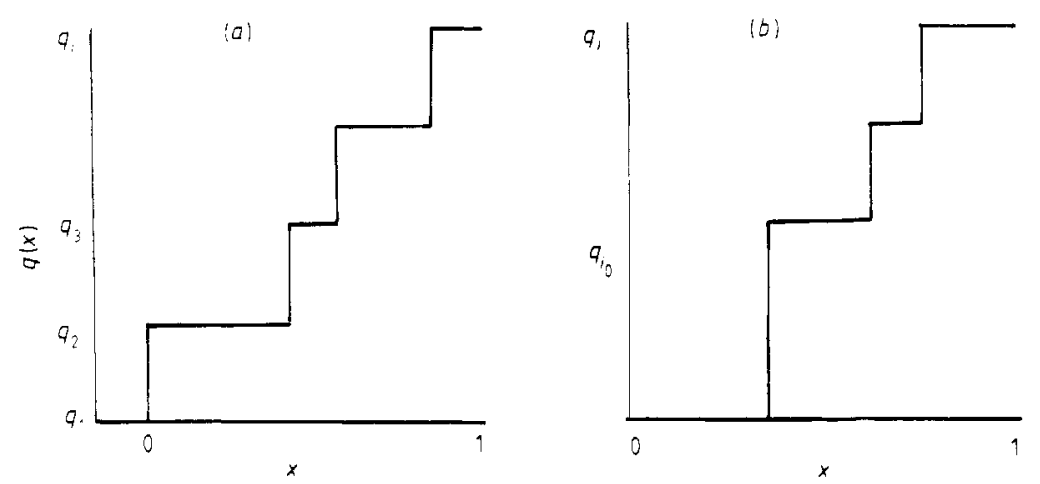

Figure 1. (a) Shape of $q(x)$ when the $a_{i}$ and $\log \alpha_{i}$ satisfy condition (16) for $T_{i-1}<T<T_{i}$. (b) Shape of $q(x)$ when the sequence $a_{i} / \log \alpha_{i}$ has a maximum for $T_{i+1}<T<T_{i}$ with $i>i_{0}$ The value of $i_{0}$ is given by formula (20).

The second case that we shall describe here is when the sequence $a_{i} / \log \alpha_{i}$ has a single maximum. So $a_{i} / \log \alpha_{i}$ increases from $1 \leqslant i \leqslant i_{1}$ and decreases for $i_{1} \leqslant i \leqslant n$. Let us call $i_{0}$ the smallest value of $i$ for which

$$
\frac{\sum_{j=1}^{i_{0}} a_{j}}{\sum_{j=1}^{i_{0}} \log \alpha_{j}}>\frac{a_{i_{0}+1}}{\log \alpha_{i_{0}+1}} .
$$

It is easy to check that $i_{0}$ is always bigger than $i_{1}$. Let us define $\theta$ and $T_{i}$ by

$$
\begin{aligned}
& \theta=\frac{J}{2}\left(\sum_{j=1}^{i_{0}} a_{j} / \sum_{j=1}^{i_{0}} \log \alpha_{j}\right)^{1 / 2} \\
& T_{i}=\frac{J}{2}\left(a_{i} / \log \alpha_{i}\right)^{1 / 2} .
\end{aligned}
$$


Then the free energy is given in the thermodynamic limit by:

if $T>\theta$

$$
\frac{1}{N} \overline{\log Z}=\log 2+J^{2} / 4 T^{2}
$$

if $\theta>T>T_{i_{0}+1}$

$$
\frac{1}{N} \overline{\log Z}=\sum_{i_{0}+1}^{n}\left(\log \alpha_{j}+a_{j} \frac{J^{2}}{4 T^{2}}\right)+\frac{J}{T}\left(\sum_{1}^{i_{0}} a_{j}\right)^{1 / 2}\left(\sum_{j=1}^{i_{0}} \log \alpha_{j}\right)^{1 / 2}
$$

if $T_{i}>T>T_{i+1}\left(\right.$ with $\left.i \geqslant i_{0}+1\right)$

$$
\begin{gathered}
\frac{1}{N} \overline{\log Z}=\sum_{i+1}^{n}\left(\log \alpha_{j}+a_{j} \frac{J^{2}}{4 T^{2}}\right)+\frac{J}{T}\left[\sum_{j=i_{0}+1}^{i}\left(a_{j} \log \alpha_{j}\right)^{1 / 2}\right] \\
+\frac{J}{T}\left(\sum_{j=1}^{i_{0}} a_{j}\right)^{1 / 2}\left(\sum_{j=1}^{i_{0}} \log \alpha_{j}\right)^{1 / 2}
\end{gathered}
$$

if $T_{n}>T$

$$
\frac{1}{N} \overline{\log Z}=\frac{J}{T}\left[\left(\sum_{j=1}^{i_{0}} a_{j}\right)^{1 / 2}\left(\sum_{j=1}^{i_{0}} \log \alpha_{j}\right)^{1 / 2}+\sum_{j=i_{0}+1}^{n}\left(a_{j} \log \alpha_{j}\right)^{1 / 2}\right] .
$$

For $T_{i}>T>T_{i+1}$, the only configurations $\nu$ which contribute to the partition function are those for which $\Sigma_{j}^{i_{0}} \varepsilon_{1}^{\nu}$ and the $\varepsilon_{j}^{\nu}$ for $i \geqslant j \geqslant i_{0}+1$ are at a finite distance from their minimal value.

$\frac{-\sum_{j=1}^{i_{0}} \varepsilon_{j}^{(\nu)}}{N J}-\left[\left(\sum_{j=1}^{i_{0}} a_{j}\right)\left(\sum_{j=1}^{i_{0}} \log \alpha_{j}\right)\right]^{1 / 2}+\frac{1}{4}\left(\sum_{j=1}^{i_{0}} a_{j} / \sum_{j=1}^{i_{0}} \log \alpha_{j}\right)^{1 / 2} \frac{\log N}{N} \simeq 0\left(\frac{1}{N}\right)$

and again the condition (19) for $i \geqslant j \geqslant i_{0}+1$.

Therefore the only possible values of $q^{\mu \nu}$ which have a finite probability in this range of temperature are $q_{i_{0}}$ and $q_{i}$ for $i_{0}+1 \leqslant j \leqslant i+1$. (See figure $1(b)$.)

There is of course no problem in making the $a_{i}$ and the $\log \alpha_{i}$ infinitesimal. All the sums in the previous formulas become integrals.

\section{A simple example}

In order to see how to calculate the free energy when $n$ is large, we will take a simple example. Let us consider the GREM whose $u$ and $v$ (see equations (10) and (12)) depend on a continuous parameter $t$ varying from 0 to 1

$$
\begin{aligned}
& u=(1-t) \log 2 \\
& v=1-(1-t)^{(2 \delta+1)}
\end{aligned}
$$

where $\delta>0$ is arbitrary. In terms of the discrete version one can set $i=n t$ and at the 
end take the limit $n \rightarrow \infty$. If $\mathrm{d} t=1 / n$ and

$$
\begin{aligned}
& \log \alpha_{i}=\log 2 \mathrm{~d} t \\
& a_{i}=(2 \delta+1)(1-t)^{2 \delta} \mathrm{d} t
\end{aligned}
$$

then the ratio $a_{i} / \log \alpha_{i}$ is a decreasing function of $i$. Since $a_{1} / \log \alpha_{1}$ is finite, there is a temperature $T_{0}$ above which the system is in its high temperature phase

$$
T_{0}=((2 \delta+1) / \log 2)^{1 / 2} J / 2
$$

and if $T>T_{0}$,

$$
N^{-1} \overline{\log Z}=\log 2+J^{2} / 4 T^{2} .
$$

Below $T_{0}$, the temperatures $T_{i}$ become dense. At a temperature $T$ below $T_{0}$, one can see that

$$
T=\frac{J}{2}\left(\frac{2 \delta+1}{\log 2}\right)^{1 / 2}(1-t)^{\delta}=T_{0}(1-t)^{\delta} .
$$

At temperature $T$, formula (18) gives

$$
\begin{aligned}
\frac{1}{N} \overline{\log Z}=\int_{t}^{1} & \left(\log 2+\frac{J^{2}}{4 T^{2}}(2 \delta+1)\left(1-t^{\prime}\right)^{2 \delta}\right) \mathrm{d} t^{\prime} \\
& +\frac{J}{T} \int_{0}^{t}[(\log 2)(2 \delta+1)]^{1 / 2}\left(1-t^{\prime}\right)^{\delta} \mathrm{d} t^{\prime} \\
= & \log 2\left\{\int_{t}^{1}\left[1+\left(\frac{T_{0}}{T}\right)^{2}\left(1-t^{\prime}\right)^{2 \delta}\right] \mathrm{d} t^{\prime}\right. \\
& \left.+\frac{2 T_{0}}{T} \int_{0}^{t}\left(1-t^{\prime}\right)^{\delta} \mathrm{d} t^{\prime}\right\} .
\end{aligned}
$$

Using (31) one gets for $T<T_{0}$,

$\frac{1}{N} \overline{\log Z}=\log 2\left[\frac{2}{1+\delta} \frac{T_{0}}{T}+\frac{2 \delta^{2}}{(2 \delta+1)(\delta+1)}\left(\frac{T}{T_{0}}\right)^{1 / \delta}\right]$.

The specific heat $C$ can then be calculated and one finds:

if $T>T_{0}$

$$
C / N=J^{2} / 2 T^{2}
$$

if $T<T_{0}$

$$
C / N=\frac{J^{2}}{2 T_{0}^{2}}\left(\frac{T}{T_{0}}\right)^{1 / \delta}
$$

Thus we see that for this simple example, the specific heat is a continuous function of temperature with a cusp at $T_{0}$. This means that the sudden freezing of the REM is replaced by a gradual freezing in the GREM due to the correlations between the energies of configurations. 


\section{The Sherrington-Kirkpatrick model and the $p$ spin-glass models}

The sK model is a system of $N$ Ising spins whose Hamiltonian $\mathscr{H}$ is given by

$$
\mathscr{H}=-\sum_{(i, j)} J_{i j} \sigma_{i} \sigma_{j} .
$$

The sum in equation (35) includes all pairs of spins in the system and the itneractions $J_{i j}$ are randomly distributed according to a distribution $\rho$

$$
\rho\left(J_{i j}\right)=\left(\frac{N}{2 \pi J^{2}}\right)^{1 / 2} \exp -\left(\frac{J_{i j}^{2} N}{2 J^{2}}\right) .
$$

It is possible to generalise the SK model by replacing the random pair interactions in equation (35) by random $\mathrm{p}$ spin interactions. The Hamiltonian of the $\mathrm{p}$ spin-glass model is (Derrida 1980)

$$
\mathscr{H}=-\sum_{i_{1} \ldots i_{p}} A_{i_{1} \ldots i_{p}} \sigma_{i_{1}} \ldots \sigma_{i_{p}} .
$$

Here again the spin $\sigma_{i}$ are Ising spins and there is an interaction $A_{i_{1} \ldots i_{p}}$ for any group of $p$ spins. The probability distribution of $A_{i_{1} \ldots i_{p}}$ is

$$
\rho\left(A_{i_{1} \ldots i_{p}}\right)=\left(\frac{N^{p-1}}{\pi J^{2} p !}\right)^{1 / 2} \exp \left(-\frac{A_{i_{1} \ldots i_{p}}^{2} N^{p-1}}{J^{2} p !}\right) .
$$

The SK model is just the case $p=2$. In the limit $p \rightarrow \infty$, one gets the REM. For $p=1$, the model reduces to $N$ non-interacting spins in a random field.

For all these $\mathrm{p}$ spin-glass models, the probability $P_{\nu}\left(E_{\nu}\right)$ that a given spin configuration $\nu$ has energy $E_{\nu}$ is given (for $N$ large or if one replaces $N^{p-1}$ in equation (38) by $(N-1) ! /(N-p) !)$ by

$$
P_{\nu}\left(E_{\nu}\right)=\left(\pi N J^{2}\right)^{-1 / 2} \exp \left(-E_{\nu}^{2} / N J^{2}\right) .
$$

This $P_{\nu}\left(E_{\nu}\right)$ is the same as the one chosen for the GREM (see equation (8)). One can also calculate $P_{\mu . \nu}\left(E_{\mu}, E_{\nu}\right)$ the probability distribution that two given spin configurations $\mu$ and $\nu$ have energies $E_{\mu}$ and $E_{\nu}$. One finds (Derrida 1981) that $P_{\mu, \nu}\left(E_{\mu}, E_{\nu}\right)$ has the form (9) with $v$ given by

$$
v=(2 t-1)^{p}
$$

where $N t$ is the number of spins $\sigma_{i}$ which are identical in configurations $\mu$ and $\nu$, that is the overlap $q^{\mu \nu}$ between the two configurations is

$$
q^{\mu \nu}=\frac{1}{N} \sum_{i=1}^{N} \sigma_{i}^{(\mu)} \sigma_{i}^{(\nu)}=2 t-1 .
$$

For a given configuration $\nu$, the number $\mathrm{e}^{N u}$ of configurations $\mu$ which have an overlap $2 t-1$ with $\nu$ is

$$
\mathrm{e}^{N u}=\frac{N !}{(N t) !(N(1-t)) !} .
$$


Therefore

$$
u=-t \log t-(1-t) \log (1-t) .
$$

When $t$ varies from $\frac{1}{2}$ to $1, v$ varies from 0 to 1 and $u$ from $\log 2$ to 0 .

There is a small difficulty in defining a GREM which has the same $u$ and $v$ as the p spinglass model. In principle when $t$ varies from 0 to 1 , the $u(t)$ given by equation (43) has an extremum at $t=\frac{1}{2}$, the $v(t)$ given by equation (40) can either have a maximum if $p$ is even or become negative if $p$ is odd. Also the overlap $q^{u v^{\prime}}$ given by equation (41) can be negative. For the GREM defined in $\S 2$, it is impossible to have a negative $v$ or to have an extremum in $u$ or in $v$ as $i$ increases. Formulae (10) and (12) show clearly that $u$ and $v$ are both positive and monotonic functions of $i$. However if we limit ourselves to the range $\frac{1}{2}<t<1$, then $u(t)$ and $v(t)$ become monotonic and positive. Then there is no difficulty in defining a GREM whose $u$ and $v$ are given by formulae (41) and (43) for $\frac{1}{2}<t<1$. We think that considering only the range $\frac{1}{2}<t<1$ for a spin-glass model means that we identify a spin configuration with the configuration where all the spins have been reversed. (If for a given spin-glass model, one makes this identification, that is the partition function is the sum of $2^{N-1}$ terms because one takes only one Boltzmann factor for a configuration and its opposite configuration, then the free energy remains the same.) One can probably modify the definition of the GREM in order to also treat the range $0<t<\frac{1}{2}$. However for the moment we do not know how to do it. So we shall study a GREM whose $u$ and $v$ are given by formulae (40) and (43) only for $\frac{1}{2}<t<1$. We shall say that this GREM has almost the same pair correlations between energies as the corresponding spin-glass model.

We will now study the GREM which has the same $u$ and $v$ as a p spin-glass model (for $\frac{1}{2}<t<1$ ). Here again since $u$ and $v$ vary continuously, the number of levels in the hierarchy must be infinite and the $a_{i}$ and $\log \alpha_{i}$ must be infinitesimal.

$$
\begin{aligned}
& a_{i}=2 p(2 t-1)^{p-1} \mathrm{~d} t \\
& \log \alpha_{i}=\log [t /(1-t)] \mathrm{d} t .
\end{aligned}
$$

To discretise the problem one must choose $i=n(2 t-1)$. So $i$ goes from 1 to $n$ when $t$ varies from $\frac{1}{2}$ to 1 .

For $p=1$ and $p=2$, the ratio $a_{i} / \log \alpha_{i}$ is a decreasing function of $i$ whereas for $p>2$, this ratio has a single maximum. So in both cases, the results given in $\S 3$ can be used.

For $p=1$, one finds that $T_{1}=x$ because the ratio $a_{i} / \log \alpha_{i} \rightarrow x$ as $t \rightarrow \frac{1}{2}$. So one finds only one phase and the free energy at temperature $T$ is given by

$$
\frac{1}{N} \overline{\log Z}=\int_{t}^{1} \mathrm{~d} t^{\prime}\left[\log \frac{t^{\prime}}{1-t^{\prime}}+\frac{2 J^{2}}{4 T^{2}}\right]+\int_{1 / 2}^{t} \mathrm{~d} t^{\prime} \frac{J}{T}\left(2 \log \frac{t^{\prime}}{1-t^{\prime}}\right)^{1 / 2}
$$

where $t$ is related to the temperature $T$ by

$$
T=\frac{J}{2}\left(\frac{2}{\log (t / 1-t)}\right)^{1 / 2}
$$

For $p=2$ (the SK case), the ratio $a_{i} / \log \alpha_{i}$ has a finite limit, 2 for $i \rightarrow 1$. Therefore there is a critical temperature given by

$$
T_{\mathrm{c}}=\frac{J}{2}\left(\lim _{i \rightarrow 1 / 2} \frac{a_{i}}{\log \alpha_{i}}\right)^{1 / 2}=\frac{J}{\sqrt{2}} .
$$


For $T>T_{c}$, the free energy is given by

$$
\frac{1}{N} \overline{\log Z}=\log 2+\frac{J^{2}}{4 T^{2}}
$$

and for $T<T_{\mathrm{c}}$

$$
\begin{aligned}
\frac{1}{N} \overline{\log Z}=\int_{t}^{1} \mathrm{~d} t^{\prime} & \left(\log \frac{t^{\prime}}{1-t^{\prime}}+\frac{4 J^{2}}{4 T^{2}}\left(2 t^{\prime}-1\right)\right) \\
& +\int_{1 / 2}^{t} \mathrm{~d} t^{\prime} \frac{J}{T}\left(4\left(2 t^{\prime}-1\right) \log \left(\frac{t^{\prime}}{1-t^{\prime}}\right)\right)^{1 / 2}
\end{aligned}
$$

where $t$ is related to $T$ by (55) in the case $p=2$.

For $p>2$, since the sequence $a_{i} / \log \alpha_{i}$ has a maximum, the critical temperature $\theta$ will be given by conditions (20) and (21)

$$
\theta=T_{\mathrm{c}}=\frac{J}{2}\left(\frac{\left(2 t_{0}-1\right)^{p}}{\log 2+t_{0} \log t_{0}+\left(1-t_{0}\right) \log \left(1-t_{0}\right)}\right)^{1 / 2}
$$

where $t_{0}$ is given by

$$
\frac{v\left(t_{0}\right)}{\log 2-u\left(t_{0}\right)}=\frac{a_{i}\left(t_{0}\right)}{\log \alpha_{i}\left(t_{0}\right)}
$$

that is, $t_{0}$ is related to $p$ by

$$
2 p=\frac{\left(2 t_{0}-1\right) \log \left[t_{0} /\left(1-t_{0}\right)\right]}{\log 2+t_{0} \log t_{0}+\left(1-t_{0}\right) \log \left(1-t_{0}\right)} .
$$

For $T>\theta$, the free energy is still given by formula (49) whereas for $T<\theta$, it is given by

$$
\begin{aligned}
\frac{1}{N} \overline{\log Z}=\frac{J}{T}[ & \left(2 t_{0}-1\right)^{p}\left(\log 2+t_{0} \log t_{0}+\left(1-t_{0}\right) \log \left(1-t_{0}\right)\right]^{1 / 2} \\
& +\frac{J}{T} \int_{t_{0}}^{t} \mathrm{~d} t^{\prime}\left[2 p\left(2 t^{\prime}-1\right)^{p-1} \log \left(t^{\prime} / 1-t^{\prime}\right)\right]^{1 / 2} \\
& +\int_{t}^{1} \mathrm{~d} t^{\prime}\left(\log \left(\frac{t^{\prime}}{1-t^{\prime}}\right)+2 p\left(2 t^{\prime}-1\right)^{p-1} \frac{J^{2}}{4 T^{2}}\right)
\end{aligned}
$$

where $t$ is related to the temperature $T$ by

$$
T=\frac{J}{2}\left(\frac{2 p(2 t-1)^{p-1}}{\log [t /(1-t)]}\right)^{1 / 2}
$$

For $p \rightarrow \infty$, one can expand the results and one finds that

$$
\theta=T_{\mathrm{c}}=\frac{J}{2(\log 2)^{1 / 2}}\left(1+\frac{4^{-p}}{2 \log 2}+\ldots\right) .
$$

The higher orders should be $4^{-2 p}$ multiplied by powers of $p$. One can also calculate the ground state $E_{\mathrm{GS}}$ using the fact that

$$
-\frac{E_{\mathrm{GS}}}{J}=\lim _{T \rightarrow 0}-T \frac{\overline{\log Z}}{N}
$$


$-\frac{E_{\mathrm{GS}}}{J}=(\log 2)^{1 / 2}\left[1-\left(2 p+\frac{1}{2 \log 2}\right) 4^{-p}\right]+(2 p)^{1 / 2} \int_{(2 p \log 2)^{1 / 2}}^{\infty} \mathrm{d} x 2 x^{2} \mathrm{e}^{-x^{2}}$.

For any spin-glass model, one can build a GREM which has (almost) the same correlations between pairs of energies. One can consider, for a given spin-glass model that the REM is a zeroth order approximation since it ignores all the correlations and that the GREM is a first-order approximation which takes into account all pair correlations but does not treat the triple correlations well.

In table 1 , we compare the properties (the ground-state energy and the critical temperature) of the REM and the GREM corresponding to the $p$ spin-glass model.

Table 1. The ground state energy $E_{\mathrm{GS}}$ and the critical temperature $T_{\mathrm{c}}$ of the REM the GREM and the $\mathrm{p}$ spinglass model for several values of $p$.

\begin{tabular}{|c|c|c|c|c|}
\hline$p$ & Quantity & REM & GREM & $p$ spin-glass model \\
\hline 1 & $-E_{\mathrm{GS}} / J$ & $(\log 2)^{1 / 2}=0.833$ & 0.7581 & $\pi^{-1 / 2}=0.5642$ \\
\hline 1 & $T_{\mathrm{c}} / J$ & $\frac{1}{2(\log 2)^{12}}=0.601$ & No transition & No transition \\
\hline $2(\mathrm{sk})$ & $-E_{\mathrm{GS}} / J$ & $(\log 2)^{1 \cdot 2}=0.833$ & 0.8246 & $=0.765$ \\
\hline $2(\mathrm{sk})$ & $T_{\mathrm{c}} / J$ & $\frac{1}{2(\log 2)^{1 / 2}}=0.601$ & $2^{-1 / 2}=0.707$ & 1 \\
\hline $\begin{array}{l}p \rightarrow \infty \\
p \rightarrow \infty\end{array}$ & $\begin{array}{l}-E_{\mathrm{GS}} / J \\
T_{\mathrm{d}} / J\end{array}$ & $\begin{array}{l}(\log 2)^{1.2}=0.833 \\
\frac{1}{2(\log 2)^{1 / 2}}=0.601\end{array}$ & $\begin{array}{l}\text { Formula (58) } \\
\left(1+\frac{4^{-p}}{2 \log 2}+\right.\end{array}$ & {$\left[1+2^{-(p-1)}\left(\frac{\pi}{p(\log 2)^{3}}\right)^{1 / 2}\right]$} \\
\hline & & & $2(\log 2)^{1 / 2}$ & $\begin{array}{c}2(\log 2)^{1 / 2} \\
(\text { Gardner } 1985)\end{array}$ \\
\hline
\end{tabular}

One sees the increase of the critical temperature and of the ground-state energy due to the correlations. However, the fact that the pair correlations are taken into account in the GREM does not change the values of $T_{\mathrm{c}}$ and $E_{\mathrm{GS}}$ much. So the GREM is a better approximation to real spin-glass models than the REM but the improvement is not spectacular. Even for $p$ large, the GREM does not seem to give the right correction to $T_{0}$ and does not present the second phase transition at low temperature (Gardner 1985).

\section{Spin-glass models on finite-dimensional lattices}

One can easily generalise the calculations in $\S 5$ to the case of a spin-glass model on a regular lattice in an arbitrary finite dimension. (The calculations done in this section are also valid for the infinite-ranged SK model.)

$$
\mathscr{H}=-\sum_{(i, j)} J_{i j} \sigma_{i} \sigma_{j}
$$

runs over the nearest neighbours on the lattice. The $J_{i j}$ are randomly distributed according to a gaussian distribution

$$
\rho\left(J_{i j}\right)=\left(\frac{z}{2 \pi J^{2}}\right)^{1 / 2} \exp -\frac{J_{i j}^{2} z}{2 J^{2}}
$$


where $z$ denotes the coordination number ( $z=2 d$ for a hypercubic lattice in dimension $d, z=N-1$ for the SK model since all the sites are neighbours). One can again calculate the probability distributions $P_{\nu}\left(E_{\nu}\right)$ and $P_{\mu, \nu}\left(E_{\mu}, E_{\nu}\right)$. One finds that

$$
P_{\nu}\left(E_{v}\right) \simeq \exp -E_{\nu}^{2} / N J^{2}
$$

as usual. (Since the normalisation in (60) has been chosen to insure this)

$$
P_{\mu, \nu}\left(E_{\mu}, E_{\nu}\right) \simeq \exp -\left(\frac{\left(E_{\mu}+E_{\nu}\right)^{2}}{4 N y J^{2}}+\frac{\left(E_{\mu}-E_{\nu}\right)^{2}}{4 N(1-y) J^{2}}\right)
$$

where $N y z / 2$ is the number of bonds which remain the same in configurations $\mu$ and $\nu$ (the bond $i j$ remains the same if $\sigma_{i}^{\mu} \sigma_{j}^{\mu}=\sigma_{i}^{\nu} \sigma_{j}^{\nu}$ ) and $N(1-y) z / 2$ is the number of bonds $i j$ such that $\sigma_{i}^{u} \sigma_{j}^{\mu}=-\sigma_{i}^{\nu} \sigma_{j}^{\nu}$. One should notice that on a finite-dimensional lattice the correlations between the energies of configurations depend on the number of bonds which change their sign and not on the number of spins flipped. So the natural distance between two-spin configurations is

$$
2 y^{\mu \nu}-1=\frac{2}{N z} \sum_{(i, j)} \sigma_{i}^{\mu} \sigma_{j}^{\mu} \sigma_{i}^{\nu} \sigma_{j}^{\nu}
$$

rather than the usual $q^{\mu \nu}=1 / N \Sigma \sigma_{i}^{\mu} \sigma_{i}^{\nu}$. In formula (63) the sum extends over all the bonds of the lattice. Of course in the infinite-range models like the sK model, $z=N-1$ and the two distances $y^{\mu \nu}$ and $q^{\mu \nu}$ are equivalent because $y^{\mu \nu}$ is related to $q^{\mu \nu}$ by $2 y^{\mu \nu}$ $-1=\left(q^{\mu \nu}\right)^{2}$. This relationship between $y^{\mu \nu}$ and $q^{\mu \nu}$ does not remain true on finitedimensional lattices.

For a given configuration $\nu$, the number $\mathrm{e}^{N_{u}}$ of configurations $\mu$ to which formula (62) applies can be expressed in terms of the properties of the pure ferromagnetic Ising model (i.e., all the $J_{i j}=1$ ) on the same lattice. If we denote by $S(\varepsilon)$ the entropy per site of the ferromagnetic model when the energy per site is $\varepsilon$, one can show that

$$
\exp N u \simeq \exp N S\left(\frac{z}{2}(1-2 y)\right) .
$$

Formula (64) is easy to understand. One wants to calculate the number of configurations $\mu$ which have $(\mathrm{Nz} / 2) y$ bonds identical to those of configuration $\nu$. Consider the pure ferromagnetic model and choose for $\nu$ the spin configuration where all the spins are $+(\sigma$ $i=+1$ for any $i)$. Then the energy per spin of the configuration $\mu$ is $z(1-2 y) / 2$. Thus the number of configurations $\mu$ is given by the entropy of the ferromagnetic model at energy $z(1-2 y) / 2$.

As in the previous section, one can consider the GREM whose $u$ and $v$ are given by

$$
\begin{aligned}
& u(y)=S\left[\frac{z}{2}(1-2 y)\right] \\
& v(y)=2 y-1 .
\end{aligned}
$$

When $y$ varies from 1 to $\frac{1}{2}, u$ varies from 0 (the entropy of an Ising ferromagnet in its ground state) to $\log 2$ (the entropy of an Ising ferromagnet at infinite temperature) and $v$ varies from 1 to 0 . As in $\S 5$, and for the same reasons, we ignore the range $0<y<\frac{1}{2}$ because $v$ becomes negative and $u$ would not be monotonic.

One can again discretise the model by choosing a GREM with $n$ steps, $i$ being related 
to $y$ by

$$
i=n(2 y-1)
$$

and since $\mathrm{d} y=1 / 2 n$, we have

$$
\begin{aligned}
& \log \alpha_{i}=z S^{\prime}(z(1-2 y) / 2) \mathrm{d} y \\
& a_{i}=2 \mathrm{~d} y .
\end{aligned}
$$

One sees in formula (68) that $\log \alpha_{i}$ is the derivative of the entropy $S$ of the ferromagnetic model with respect to $y$ which is related to the energy. Therefore this derivative is nothing but the inverse temperature of the ferromagnetic model. If we define the function $\tau(\varepsilon)$ by: $\tau(\varepsilon)$ is the temperature of the pure ferromagnetic model when the energy per spin is $\varepsilon$, then one has

$$
\log \alpha_{i}=z\left\{\tau\left[\frac{z}{2}(1-2 y)\right]\right\}^{-1} \mathrm{~d} y
$$

since in a pure ferromagnetic model, one expects a second-order phase transition, the temperature is a continuous and increasing function of energy. Therefore the ratio $a_{i} / \operatorname{lgg} \alpha_{i}$ is a continuous and decreasing function of $y$ and $i$. So we are in the first case described in $\S 3$ and we can use formulas (17) and (18) to solve the GREM. Since when $y \rightarrow \frac{1}{2}, \tau \rightarrow \infty$, the temperature $T_{1}$ is infinite. When the GREM is at a given temperature $T$, one can calculate the value of $y$ corresponding to this temperature by using equation (17)

$$
T=\frac{J}{2}\left\{\frac{2}{z} \tau\left[\frac{z}{2}(1-2 y)\right]\right\}^{1 / 2}
$$

and once $y$ is known from (71), one gets for the average free energy

$$
\begin{aligned}
\frac{1}{N} \overline{\log Z}=\int_{y}^{1} \mathrm{~d} y^{\prime}\left(\frac{z}{\tau\left[(z / 2)\left(1-2 y^{\prime}\right)\right]}+\frac{2 J^{2}}{4 T^{2}}\right) \\
+\int_{1 / 2}^{y} \mathrm{~d} y^{\prime} \frac{J}{T}\left(2 \frac{z}{\tau\left[(z / 2)\left(1-2 y^{\prime}\right)\right]}\right)^{1 / 2} .
\end{aligned}
$$

This is the free energy of the GREM which has the same pair correlations between energies ( for $\frac{1}{2}<y<1$ ) as a given spin-glass model on any regular lattice.

One can rewrite formula (72) in a simpler form. If we call $C(\tau)$ the specific heat per spin of the ferromagnetic system on the same lattice, then formula (72) becomes

$$
\frac{1}{N} \overline{\log Z}=\int_{0}^{\tau} \mathrm{d} \tau_{1} C\left(\tau_{1}\right)\left(\frac{1}{\tau}+\frac{1}{\tau_{1}}\right)+\int_{\tau}^{\infty} \mathrm{d} \tau_{1} C\left(\tau_{1}\right) \frac{2}{\left(\tau \tau_{1}\right)^{1 / 2}}
$$

where $\tau$ is related to the temperature $T$ of the GREM by

$$
\tau=2 z(T / I)^{2} .
$$

We see that if we know the properties of a ferromagnetic model on a given lattice, we know the free energy of the GREM which has the same pair correlations as the spin glass model on this lattice.

Formula (73) remains valid in the case of the SK model studied in $\S 5$. It is also valid for the $\mathrm{p}$ spin-glass models with the convention that when the ferromagnetic model has 
a first-order phase transition ( for $p>2$ ), the latent heat is represented by a delta function in the specific heat $C(\tau)$.

From (74), one sees that the GREM has a critical temperature $T_{\mathrm{c}}$ given by

$$
T_{\mathrm{c}}=J\left(\tau_{\mathrm{c}} / 2 z\right)^{1 / 2}
$$

where $\tau_{\mathrm{c}}$ is the critical temperature of the ferromagnetic model. Using formula (73), one can easily show that if the specific heat $C(\tau)$ has a critical exponent $\alpha$, then the specific heat of the GREM has a critical exponent $\alpha-1$.

$$
[C(\tau)]_{\text {sing }} \simeq\left|\tau-\tau_{\mathrm{c}}\right|^{-\alpha} \Rightarrow\left[C_{\mathrm{GREM}}(T)\right]_{\text {sing }} \simeq\left|T-T_{\mathrm{c}}\right|^{1-\alpha} .
$$

We believe that the rule $\alpha \rightarrow \alpha-1$ is a consequence of the approximation made by using the GREM instead of the true spin-glass model. One knows for example in dimension two that the ferromagnet has a phase transition whereas most of the studies done up to now indicate that there is no spin-glass phase at finite temperature in $d=2$. However the rule $\alpha \rightarrow \alpha-1$ indicates (at least as an approximation) that the specific heat of a spin glass cannot diverge (since $\alpha$ is always less than 1 ).

\section{The infinite-range Potts glass}

The infinite-range Potts glass has been a controversial subject in the recent past. There was a discussion over whether the Parisi approach could be used in the case of the Potts glass (Elderfield and Sherrington 1983, Elderfield 1984, Lage and Erzan 1983, Lage and Nunes da Silva 1984, Nishimori and Stephen 1983). At the moment, the difficulty seems to have been overcome by Gross et al (1985) who claim that there are two phase transitions when the number of states is large enough.

For the infinite-range Potts model, one can again calculate the correlation between energy levels. The $\mathrm{q}$ state Potts glass can be defined by the following Hamiltonian

$\mathscr{H}=-\sum_{i<j} \sum_{K=1}^{q-1} G_{i j}^{(K)} \cos \left(\frac{2 \pi K}{q}\left(p_{i}-p_{j}\right)\right)+H_{i j}^{(K)} \sin \left(\frac{2 \pi K}{q}\left(p_{i}-p_{j}\right)\right)$

where the sum extends over all pairs of spins $(1 \leqslant i<j \leqslant N)$ and $p_{i}$ denotes the value of the spin at site $i\left(1 \leqslant p_{i} \leqslant q\right)$.

The $G_{i j}^{K}$ and $H_{i j}^{K}$ are randomly distributed according to a given probability distribution

$$
\begin{aligned}
& \rho\left(G_{i j}^{(K)}\right) \simeq \exp \left(-\frac{\left(G_{i j}^{(K)}\right)^{2}}{2 J^{2}} N(q-1)\right) \\
& \rho\left(H_{i j}^{(K)}\right) \simeq \exp \left(-\frac{\left(H_{i j}^{(K)}\right)^{2}}{2 J^{2}} N(q-1)\right) .
\end{aligned}
$$

Obviously in the case of the q state Potts model, the total number of configurations is no longer $2^{N}$ but becomes $q^{N}$.

One can again, calculate the probability distributions $P_{\nu}\left(E_{\nu}\right)$ and $P_{\mu, \nu}\left(E_{\mu}, E_{\nu}\right)$

$$
P_{\nu}\left(E_{\nu}\right) \simeq \exp -E_{\nu}^{2} / N J^{2}
$$

$P_{\mu, \nu}\left(E_{\mu}, E_{\nu}\right) \simeq \exp \left[-\frac{1}{2 N J^{2}}\left(\frac{\left(E_{\mu}+E_{\nu}\right)^{2}(q-1)}{q(s+1)-2}+\frac{\left(E_{\mu}-E_{\nu}\right)^{2}(q-1)}{q(1-s)}\right)\right]$

where $s$ is defined in the following way: $N^{2} s / 2$ is the number of bonds $i j$ whose energy is 
the same in configurations $\mu$ and $\nu$, that is the number of bonds $i, j$ such that $p_{i}^{(\nu)}-$ $p_{j}^{(\nu)}=p_{i}^{(\mu)}-p_{i}^{(u)}$. If $N_{K}=N x_{K}$ is the number of sites for which $p_{l}^{(u)}=p_{l}^{(\nu)}+K(\bmod q)$ for $0 \leqslant K \leqslant q-1$, then one has

$$
\frac{N^{2} s}{2}=\sum_{K=0}^{q-1} \frac{N_{K}^{2}}{2}=\frac{N^{2}}{2} \sum_{K=0}^{q-1} x_{K}^{2}
$$

One can also calculate the number $\mathrm{e}^{v_{u}}$ of configurations $\mu$ to which formula (80) applies

$$
\mathrm{e}^{N_{u}}=\sum_{\left\{N_{K}\right\}} \frac{N !}{N_{1} ! \ldots N_{q-1} !}
$$

where in formula (82) the $N_{K}$ are constrained to satisfy formula (81). For a given value of $s$, the sum (82) is dominated by the region where one of the $N_{K}$, say $N_{0} \simeq N x$ with $x>1 / q$, all the others being equal to $N(1-x) /(q-1)$. Therefore when

$$
s=x^{2}+(1-x)^{2} /(q-1)
$$

one has

$$
u=-x \log x-(1-x) \log [(1-x) /(q-1)] .
$$

By comparing formulae (80) and (9) one sees that one can build a GREM whose $u$ and $v$ are given by formulae (84) and (85)

$$
v=[(x q-1) /(q-1)]^{2} \text {. }
$$

When $x$ varies from $1 / q$ to $1, v$ goes from 0 to 1 and $u$ from $\log q$ to 0 . One finds that as for the p spin-glass model, the ratio $a_{i} / \log \alpha_{i}$ has a single maximum when $q>2$. One can again use the results of $\S 3$. This GREM has a phase transition at a critical temperature $T_{\mathrm{c}}$ given by

$$
T_{\mathrm{c}}=\theta=\frac{J}{2}\left(\frac{q(q-2)}{(q-1)^{2} \log (q-1)}\right)^{1 / 2} .
$$

For $T>T_{\mathrm{c}}$, one has

$$
\frac{1}{N} \overline{\log Z}=\log q+\frac{J^{2}}{4 T^{2}}
$$

whereas for $T<T_{\mathrm{c}}$

$$
\begin{aligned}
\frac{1}{N} \overline{\log Z}=\frac{J}{T}( & \left.\frac{(q-2)^{3} \log (q-1)}{q(q-1)^{2}}\right)^{1 / 2} \\
& +\frac{J}{T} \int_{(q-1) / q}^{x} \mathrm{~d} x_{1}\left[\frac{2 q\left(q x_{1}-1\right)}{(q-1)^{2}} \log \left(\frac{x_{1}(q-1)}{1-x_{1}}\right)\right]^{1 / 2} \\
& +\frac{J^{2}}{4 T^{2}}\left[1-\left(\frac{x q-1}{q-1}\right)^{2}\right]-x \log x-(1-x) \log \left(\frac{1-x}{q-1}\right)
\end{aligned}
$$

where $x$ is related to the temperature by

$$
T=\frac{J}{2}\left\{\frac{2 q(x q-1)}{(q-1)^{2}}\left[\log \left(\frac{x(q-1)}{1-x}\right)\right]^{-1}\right\}^{1 / 2} .
$$


Again, the GREM does not seem to have any trace of the second transition predicted by Gross et al (1985) for the q state Potts glass.

\section{The correlations between three energy levels}

We have seen in $\S \S 5,6$ and 7 that one can build a GREM which has (almost) the same $P_{v}$ $\left(E_{\nu}\right)$ and $P_{\mu, \nu}\left(E_{\mu}, E_{\nu}\right)$ as a given spin-glass model. Looking at table 1 it can be clearly seen that a spin-glass model and the corresponding GREM do not have the same properties. It is therefore interesting to understand why their properties differ.

We think that the origin of the difference is rather obvious. A spin-glass model and the corresponding GREM have no reason to have the same three energy correlations or higher correlations. To see that, let us calculate the probability $P_{\lambda, \mu . \nu}\left(E_{\lambda}, E_{\mu}, E_{\nu}\right)$ for the $p$ spin-glass model and for the corresponding GREM.

Let us first calculate $P_{\lambda, \mu . \nu}$ in the case of the GREM. Consider that configurations $\lambda$ and $\mu$ are in the same group for $j \leqslant i$ and in different ones for $j>i$ and that configurations $\lambda$ and $\nu$ are in the same group for $j \leqslant i^{\prime}$ and different groups for $j>i^{\prime}$ and that $i^{\prime} \geqslant i$. This means that $q^{\lambda \mu}=q_{i}$ and $q^{\lambda \nu}=q_{i}$, with $q^{\lambda \mu} \leqslant q^{\lambda \nu}$. Because of the ultrametric structure of the model, one has $q^{\mu \nu}=q_{i}=q^{\lambda \nu}$. So $P_{\lambda, \mu . \nu}$ depends only on two parameters $v_{1}$ and $v_{2}$,

$$
\begin{aligned}
& v_{1}=\sum_{j=1}^{i-1} a_{j} \\
& v_{2}=\sum_{j=1}^{i-1} a_{j} .
\end{aligned}
$$

One can of course write

$$
\begin{aligned}
& E_{i}=e_{\lambda}+\rho+\varphi \\
& E_{\mu}=e_{\mu}+\varphi \\
& E_{\nu}=e_{\nu}+\rho+\varphi
\end{aligned}
$$

where

$$
\begin{array}{ll}
\varphi=\sum_{j=1}^{i-1} \varepsilon_{j}^{(\lambda)} & \rho=\sum_{j=i}^{i^{\prime}-1} \varepsilon_{j}^{(\lambda)} \quad e_{i}=\sum_{j=i^{\prime}}^{n} \varepsilon_{j}^{(\lambda)} \\
e_{\mu}=\sum_{j=i}^{n} \varepsilon_{j}^{(\mu)} & e_{\nu}=\sum_{j=i^{\prime}}^{n} \varepsilon_{j}^{(\nu)} .
\end{array}
$$

These five variables are gaussian and their widths can be expressed in terms of $v_{1}$ and $v_{2} . P_{\lambda, \mu, \nu}\left(E_{i}, E_{\mu}, E_{\nu}\right)$ is given by

$$
P_{j, \mu \nu}\left(E_{\lambda}, E_{\mu}, E_{\nu}\right)=\int \mathrm{d} \varphi \mathrm{d} \rho \exp -h\left(E_{\lambda}, E_{\mu}, E_{\nu}, \rho, \varphi\right)
$$

where

$$
h=\frac{1}{N J^{2}}\left(\frac{\varphi^{2}}{v_{1}}+\frac{\rho^{2}}{v_{2}-v_{1}}+\frac{\left(E_{\mu}-\varphi\right)^{2}}{1-v_{1}}+\frac{\left(E_{\dot{\lambda}}-\rho-\varphi\right)^{2}+\left(E_{v}-\rho-\varphi\right)^{2}}{1-v_{2}}\right) .
$$


The integral in formula (93) is easy to do and one finds that

$$
P_{\lambda, \mu, \nu}\left(E_{\lambda}, E_{\mu}, E_{\nu}\right) \simeq \exp -\frac{1}{N J^{2}} g\left(E_{\lambda}, E_{\mu}, E_{\nu}\right)
$$

where $g$ is given for the GREM by

$$
\begin{aligned}
g_{\text {GREM }}\left(E_{\lambda}, E_{\mu}, E_{\nu}\right)= & \frac{1}{2\left(1-v_{2}\right)}\left(E_{\lambda}-E_{\nu}\right)^{2} \\
& +\frac{1}{1+v_{2}-2 v_{1}^{2}}\left(\frac{\left(E_{\lambda}+E_{\nu}\right)^{2}}{2}+E_{\mu}^{2}\left(1+v_{2}\right)-2 E_{\mu}\left(E_{\lambda}+E_{\nu}\right) v_{1}\right) .
\end{aligned}
$$

We see that $g$ depends only on two parameters $v_{1}$ and $v_{2}$ because of the ultrametric structure of the model.

Let us now calculate $P_{\lambda, \mu, \nu}$ for the p spin-glass model defined by the Hamiltonian (37). $P_{\lambda, \mu, \nu}$ is given by

$$
P_{i, \mu, \nu}=\left\langle\delta\left[E_{\lambda}-\mathscr{H}\left(\sigma_{i}^{\lambda}\right)\right] \delta\left[E_{\mu}-\mathscr{H}\left(\sigma_{i}^{\mu}\right)\right] \delta\left[E_{\nu}-\mathscr{H}\left(\sigma_{i}^{\nu}\right)\right]\right\rangle .
$$

By using the representation $\delta(x)=(1 / 2 \pi) \int \mathrm{e}^{i x \alpha} \mathrm{d} \alpha$ and the fact that the averages are done on Gaussian variables, one can easily show that $P_{\lambda, \mu, \nu}$ has form (95) with

$$
\begin{aligned}
g_{\substack{\text { sin } \\
\text { glass }}}\left(E_{\lambda}, E_{\mu},\right. & \left.E_{\nu}\right)=\frac{1}{1-\left(q_{\lambda \mu}^{2 p}+q_{\nu \lambda}^{2 p}+q_{\mu \nu}^{2 p}\right)+2 q_{\lambda \mu}^{p} q_{\lambda \nu}^{p} q_{\mu \nu}^{p}} \\
& \times\left[E_{\lambda}^{2}\left(1-q_{\mu \nu}^{2 p}\right)+E_{\mu}^{2}\left(1-q_{\lambda \nu}^{2 p}\right)+E_{\nu}^{2}\left(1-q_{\mu \lambda}^{2 p}\right)\right. \\
& +2 E_{\lambda} E_{\mu}\left(q_{\lambda \nu}^{p} q_{\mu \nu}^{p}-q_{\lambda \mu}^{p}\right)+2 E_{\lambda} E_{\nu}\left(q_{\lambda \mu}^{p} q_{\mu \nu}^{p}-q_{\lambda \nu}^{p}\right) \\
& \left.+2 E_{\mu} E_{\nu}\left(q_{\lambda \mu}^{p} q_{\lambda \nu}^{p}-q_{\mu \nu}^{p}\right)\right] .
\end{aligned}
$$

One sees that formula (98) depends on three parameters $q_{\lambda \mu}, q_{\lambda \nu}$ and $q_{\mu \nu}$ and so differs from (96). However if one tries to make a comparison by choosing $q_{\mu \nu}=q_{\lambda \nu}$ as in the GREM and by defining (see equations (40)-(41))

$$
v_{1}=q_{\lambda \mu}^{p_{\lambda}}=q_{\mu \nu}^{p} \quad \text { and } \quad v_{2}=q_{\lambda \nu}^{p}
$$

then one finds that formula (98) becomes identical to (96).

Sn we see that in the p spin-glass model and in the corresponding GREM, the triple correlations are in general different because in the $\mathrm{p}$ spin-glass model, they depend on three parameters whereas in the GREM they depend only on two parameters. However when one chooses $q_{i, \mu}=q_{\mu \nu}$ in the p spin-glass model the correlations become identical to those of the GREM. So we think that the GREM does not distort the triple correlations too much.

\section{Conclusion}

In this paper we have obtained the solution of the GREM. We have seen that for any spinglass model, one can build a GREM which has the same pair correlations between energies (with the restriction discussed in $\S 4$ ).

Our main result is formula (73) which is valid for all the cases that we have considered. It gives, for each spin glass model on a given lattice, the free energy of the corresponding 
GREM in terms of the specific heat of the pure ferromagnetic Ising model on the same lattice.

These generalised random energy models provide a whole class of spin-glass models which can be solved exactly. We have seen that when the correlations between the energies are those of a spin-glass model, the specific heat exponent $\alpha$ of the GREM at the transition cannot be positive. This agrees with the fact that one expects that the specific heat of a spin glass does not diverge. There remain interesting questions to be answered for the GREM: is it possible to calculate the function $q(x)$ (De Dominicis and Hilhorst 1985 ), to study non-self-averaging effects and to define dynamics for the GREM (De Dominicis et al 1985, De Dominicis 1985).

We have seen that the GREM can be viewed as an approximation to any spin-glass model. This approximation is better than the RE approximation but the improvement is not spectacular. For the $\mathrm{p}$ spin-glass models and for the Potts glass, the GREM does not show the second-phase transition predicted by the replica calculations. For the p spinglass model, in the limit $p \rightarrow \infty$, the GREM does not give the first correction which can be calculated using replicas.

The GREM takes into account pair correlations between energies. It would be interesting to see whether a hierarchy of approximations to spin-glass models could be developed which would take into account two energy correlations, three energy correlations, etc. It would also be interesting to introduce a magnetic field in the GREM, to calculate the magnetic susceptibility and the de Almeida Thouless line (de Almeida and Thouless 1978).

Lastly we think that it would be worthwhile to consider distributions of the interactions which are not Gaussian. For example if $\rho\left(J_{i j}\right)$ is given by

$$
\rho\left(J_{i j}\right)=\frac{1}{2}\left[\delta\left(J_{i j}+J\right)+\delta\left(J_{i j}-J\right)\right]
$$

then formula (61) becomes

$$
P_{\nu}\left(E_{\nu}\right) \simeq \exp -N f(E / N J)
$$

where

$$
f(x)=\frac{x}{2} \log \left(\frac{z+2 x}{z-2 x}\right)+\frac{z}{4} \log \left(1-\frac{4 x^{2}}{z^{2}}\right)
$$

and $z$ is the coordination number. Formula (62) becomes

$P_{\mu, \nu}\left(E_{\mu}, E_{\nu}\right) \simeq \exp \left[-N y f\left(\frac{\left(E_{\mu}+E_{\nu}\right)}{2 N J y}\right)-N(1-y) f\left(\frac{\left(E_{\mu}-E_{\nu}\right)}{2 N J(1-y)}\right)\right]$

\section{Acknowledgments}

We would like to thank $\mathrm{C}$ de Dominicis and $\mathrm{H}$ J Hilhorst for useful discussions.

Financial support for one of us (EG) was from the Science and Engineering Research Council, UK.

\section{Appendix}

The free energy of the generalised random energy model for the two cases considered 
in $\S 3$ (equations (18) and (23)) can be derived by a generalisation of the proof for two stages (Derrida 1985) to an arbitrary number of stages.

Firstly the expectation of the number of largest groups with energies $\varepsilon_{1}$ between $\varepsilon_{1}$ and $\varepsilon_{1}+\mathrm{d} \varepsilon_{1},\left\langle\hat{N}_{1}\left(\varepsilon_{1}\right)\right\rangle \mathrm{d} \varepsilon_{1}$, is calculated. $\mathrm{d} \varepsilon_{1}$ must be smaller than $N^{\alpha}$ with $\alpha<1$ in order to have a well defined thermodynamic limit. $\left\langle\mathcal{N}_{1}\left(\varepsilon_{1}\right)\right\rangle$ behaves as $\exp N g_{1}\left(\varepsilon_{1}\right)$. If $g_{1}\left(\varepsilon_{1}\right)<0$, then for almost all systems $\mathcal{N}_{1}\left(\varepsilon_{1}\right)=0$ and there is only a small probability which vanishes exponentially with $N$ that $\hat{N}_{1}\left(\varepsilon_{1}\right) \geqslant 1$. If $g_{1}\left(\varepsilon_{1}\right)>0$, then since the energies $\varepsilon_{1}$ of the groups are independent random variables, $\mathcal{N}_{1}\left(\varepsilon_{1}\right)$ is equal to its expectation with a variance proportional to the expectation. Therefore if $g_{1}\left(\varepsilon_{1}\right)>0, \mathcal{N}_{1}\left(\varepsilon_{1}\right) \simeq$ $\left\langle\hat{N}_{1}\left(\varepsilon_{1}\right)\right\rangle . \hat{N}_{1}\left(\varepsilon_{1}\right)$ can then be used to calculate the number $\hat{N}_{2}\left(E_{2}\right)$ of next largest groups with energies $E_{2}$ where $E_{2}=\varepsilon_{1}+\varepsilon_{2}$ by repeating the above argument. This process can be iterated to calculate $\mathcal{N}_{n}(E)$ the number of smallest groups with energy $E$. The logarithm of this number is the entropy and this will be used to derive the free energy. At the $i$ th stage, let $E_{i}=\sum_{k=1}^{i} \varepsilon_{k}$ and the number of groups with energy $E_{i}$ be $\mathscr{N}_{i}\left(E_{i}\right)$.

Let us define $f_{i}\left(E_{i}\right)$ by

$$
\mathcal{N}_{i}\left(E_{i}\right) \simeq \exp N f_{i}\left(E_{i}\right)
$$

where $\mathfrak{N}_{i}\left(E_{i}\right)$ denotes the typical (or most probable) value of $\mathcal{N}_{i}\left(E_{i}\right)$. We are going to calculate $f_{i}\left(E_{i}\right)$ in different cases. We shall only consider the negative $E_{i}$ because $f_{i}\left(E_{i}\right)$ is symmetric and only negative energies play a role in the calculation of the free energy.

\section{A1. First case}

Let us first consider the case where the sequence $a_{j} / \log \alpha_{j}$ is decreasing for $1 \leqslant j \leqslant i$.

$$
\frac{a_{1}}{\log \alpha_{1}}>\frac{a_{2}}{\log \alpha_{2}}>\ldots>\frac{a_{i}}{\log \alpha_{i}} \text {. }
$$

Then we shall prove by induction that

$$
f_{i}\left(E_{i}\right)=\sum_{\kappa=j+1}^{i} \log \alpha_{\kappa}-\frac{\left[E_{i}+N J \sum_{k=1}^{i}\left(a_{k} \log \alpha_{k}\right)^{1 / 2}\right]^{2}}{N^{2} J^{2} \sum_{\kappa=j+1}^{i} a_{\kappa}}
$$

where $j$ is defined by

$$
N J e_{i-j+1}^{i}<\left|E_{i}\right|<N J e_{i-j}^{i} \quad \text { for } j=0, \ldots, i-1
$$

where

$$
\begin{array}{rlrl}
e_{i-j}^{i}=\sum_{k=j+1}^{i} & \frac{a_{k}\left(a_{j+1} \log \alpha_{j+1}\right)^{1 / 2}}{a_{j+1}} & & \\
& +\sum_{k=1}^{j}\left(a_{\kappa} \log \alpha_{k}\right)^{1 / 2} & & \text { for } 1 \leqslant j \leqslant i-1 \\
= & \sum_{k=1}^{i} a_{\kappa}\left(\frac{\log \alpha_{j+1}}{a_{j+1}}\right)^{1 / 2} & & \text { for } j=0 \\
=0 & \text { for } j=-1
\end{array}
$$

and

$$
f_{i}\left(E_{i}\right)=-\infty \quad \text { if }\left|E_{i}\right|>N J e_{1}^{i} .
$$


One can easily check that formulae (A3)-(A6) are true for $i=1$ since $f_{1}\left(E_{1}\right)=\log \alpha_{1}$ $-\left(E_{1}^{2} / N^{2} J^{2} a_{1}\right)$ which is positive if and only if $\left|E_{1}\right| / N J<\left(a_{1} \log \alpha_{1}\right)^{1 / 2}$. Let us assume that formulae (A3)-(A6) are true at the $i$ th stage, then $\hat{N}_{1+1}\left(E_{i+1}\right)$ at the $i+1$ th stage is given by

$$
\hat{N}_{i+1}\left(E_{i+1}\right)=\int \mathrm{d} E_{i} \wedge\left(E_{i}\right) \exp N\left(\log \alpha_{i+1}-\frac{\left(E_{i+1}-E_{i}\right)^{2}}{N^{2} J^{2} a_{i+1}}\right) .
$$

This integral can be done using the saddle point method (since $N$ is large)

$$
\frac{\log N_{i+1}\left(E_{i+1}\right)}{N}=\max _{E_{i}}\left(f_{i}\left(E_{i}\right)+\log \alpha_{i+1}-\frac{\left(E_{i+1}-E_{i}\right)^{2}}{N J^{2} a_{i+1}}\right) .
$$

The value of $E_{i}$ which will give the maximum of $(\mathrm{A} 8)$ depends on $E_{i+1}$. If one chooses $E_{i+1}$ such that this maximum is in the following interval

$$
e_{i-j+1}^{i}<\left|E_{i}\right| / N J<e_{i-j}^{i}
$$

then $E_{i}$ is given by

$$
E_{i}=\frac{E_{i+1} \Sigma_{k=j+1}^{i} a_{k}-a_{i+1} \Sigma_{k=1}^{j}\left(a_{k} \log \alpha_{k}\right)^{1 / 2}}{\sum_{k=j+1}^{i+1} \alpha_{k}} N J
$$

and if we substitute this expression in (A8), then

$$
f_{i+1}\left(E_{i+1}\right)=\sum_{\kappa=j+1}^{i+1} \log \alpha_{k}-\frac{\left(E_{i+1}+\sum_{\kappa=1}^{j}\left(a_{k} \log \alpha_{\kappa}\right)^{1 / 2} N J\right)^{2}}{\sum_{\kappa=j+1}^{i+1} a_{\kappa}} .
$$

The condition on $E_{i+1}$ which insures (A9) can easily be obtained from (A9) and (A10)

$$
e_{i-j+2}^{i+1}<\left|E_{i+1}\right| / N J<e_{i-j+1}^{i+1} \quad 0 \leqslant j \leqslant i-1 .
$$

We have now to consider the case where $\left|E_{i+1}\right| / N J>e_{2}^{i+1}$. In this case the maximum in (A8) is given by the end point $\left|E_{i}\right| / N J=e_{1}^{i}$ and again the maximum value of (A8) is given by (A11). Formula (A11) is again valid only when $f_{i+1}\left(E_{i+1}\right)$ is positive that is, when $\left|E_{i+1}\right| / N J<e_{1}^{i+1}$. When the maximum value of (A8) becomes negative, then $\hat{N}_{i+1}\left(E_{i+1}\right)=0$ with probability 1 and therefore $f_{i+1}\left(E_{i+1}\right)=-\infty$ for $\mid E_{i+1} / N J>e_{1}^{i+1}$. This proves formulae (A3)-(A6) when the condition (A2) is satisfied.

\section{A2. Second case}

Let us now consider the case where the sequence $a_{i} / \log \alpha_{i}$ satsifies

$$
\frac{a_{j+1}}{\log \alpha_{j+1}}>\frac{\sum_{k=1}^{j} a_{k}}{\sum_{k=1}^{j} \log \alpha_{k}} \quad \text { for } 1 \leqslant j \leqslant i .
$$

This condition implies that $a_{2} / \log \alpha_{2}>a_{1} / \log \alpha_{1}$ but does not mean that the sequence $a_{i} / \log \alpha_{i}$ is increasing. If condition (A12) is satisfied, we shall show that

$$
f_{i}\left(E_{i}\right)=\sum_{k=1}^{i} \log \alpha_{k}-\frac{E_{i}^{2}}{N J^{2}\left(\sum_{k=1}^{i} a_{k}\right)}
$$


for

$$
\left|E_{i}\right|<N J\left[\left(\sum_{k=1}^{i} a_{k}\right)\left(\sum_{k=1}^{i} \log \alpha_{k}\right)\right]^{1 / 2}
$$

and

$$
f_{i}\left(E_{i}\right)=-\infty \quad \text { for }\left|E_{i}\right|>N J\left[\left(\sum_{k=1}^{i} a_{k}\right)\left(\sum_{k=1}^{i} \log \alpha_{k}\right)\right]^{1 / 2}
$$

Again it is easy to prove formulae (A13) and (A14) by induction. For $i=1$, it is correct. If one asumes that it is valid for the $i$ th stage, one can prove that it remains valid for the $i+1$ th stage. One has again

$$
f_{i+1}\left(E_{i+1}\right)=\max _{E_{i}}\left(f_{i}\left(E_{i}\right)+\log \alpha_{i+1}-\frac{\left(E_{i+1}-E_{i}\right)^{2}}{N J^{2} a_{i+1}}\right)
$$

when this maximum value is positive and $f_{i+1}\left(E_{i+1}\right)=-\infty$ otherwise. If (A14) is satisfied, one finds that $E_{i}$ which makes (A16) maximum is given by

$$
E_{i}=\frac{\sum_{x=1}^{i} a_{k}}{\sum_{k=1}^{i+1} a_{k}} E_{i+1} \text {. }
$$

Using the fact that now

$$
\frac{a_{i+1}}{\log \alpha_{i+1}}>\frac{\sum_{k=1}^{i} a_{k}}{\sum_{k=1}^{i} \log \alpha_{k}}
$$

One finds that as long as $f_{i+1}\left(E_{i+1}\right)$ is positive, $E_{i}$ satisfies (A14). Therefore, the only limitation on $E_{i+1}$ is that $f_{i+1}\left(E_{i+1}\right)$ must be positive which can be expressed as

$$
\left|E_{i+1}\right|<N J\left[\left(\sum_{k=1}^{i+1} a_{k}\right)\left(\sum_{k=1}^{i+1} \log \alpha_{k}\right)\right]^{1 / 2} .
$$

\section{A3. Third case}

We can consider the case where $a_{i} / \log \alpha_{i}$ has a maximum. So this sequence will satisfy (A12) for $i \leqslant i_{0}-1$ and for $i \geqslant i_{0}$ the sequence is decreasing. (If $i_{1}$ is the value of $i$ for which $a_{i} / \log \alpha_{i}$ is maximum, then $i_{0}$ is always larger than $i_{1}$.) For $i \leqslant i_{0}-1$, then $f_{i}\left(E_{i}\right)$ is given by (A13). For $i=i_{0}(\mathrm{~A} 12)$ is identical to (A3). And from $i>i_{0}$, one can again give a proof by induction and find that (A3)-(A13) are valid provided that $a_{1}$ and $\log \alpha_{1}$ are replaced by $\sum_{k=1}^{i_{0}} a_{k}$ and by $\sum_{k=1}^{i_{0}=1} \log \alpha_{k}$ and that $a_{i+1}$ and $\log \alpha_{i+1}$ are replaced by $a_{i+i_{0}}$ and $\log \alpha_{i+i_{0}}$.

\section{A4. The free energy}

Expressions (18) and (23) for the free energy can then be derived easily. The entropy $S(E)$ of the model at energy $E$ is equal to the logarithm of the number of configurations:

$$
S(E)=N f_{n}(E) \text {. }
$$

In order to convert $S(E)$ into the free energy $\overline{\log Z}$ one uses the fact that

$$
\frac{\mathrm{d} S}{\mathrm{~d} E}=T^{-1}
$$


where $T$ is the temperature and that

$$
\overline{\log Z}=-T^{-1} E+S .
$$

The values $N J e_{n-j}$ where the function $f_{n}(E)$ changes its shape give rise to the temperatures $T_{i}$.

\section{References}

de Almeida J R L and Thouless D J 1978 J. Phys. A: Math. Gen. 11983

Blandin A, Gabay M and Garel T 1980 J. Phys. C: Solid State Phys. 13403

Chowdhury D and Mookerjee A 1984 Phys. Rep. 1141

De Dominicis C and Hilhorst H J 1985 J. Physique Lett. 46909

De Dominicis C, Orland H and Lainee F 1985 J. Physique Lett. 46463

De Dominicis C 1985 International Conference on Magnetism, San Francisco

Derrida B 1980 Phys. Rev. Lett. 4579

— 1981 Phys. Rev. B 242613

- 1985 J. Physique Lett. 46401

Derrida B and Toulouse G 1985 J. Physique Lett. 46223

Elderfield D 1984 J. Phys. A: Math. Gen. 17 L517

Elderfield D and Sherrington D 1983 J. Phys. C: Solid State Phys. 16 L497, 471 and 1159

Gardner E 1985 Nucl. Phys. B [FS14] 747

Gross D J, Kantor I and Sompolinsky H 1985 Phys. Rev. Lett. 55304

Gross D J and Mezard M 1984 Nucl. Phys. B 240 (FS12) 431

Lage E J S and Erzan A 1983 J. Phys. C: Solid State Phys. 16 L873

Lage E J S and Nunes da Silva J M 1984 J. Phys. C: Solid State Phys. 17 L593

Mezard M, Parisi G, Sourlas N, Toulouse G and Virasoro M 1984a Phys. Rev. Lett. 521146 1984b J. Physique $\mathbf{4 5} 843$

Mezard M, Parisi G and Virasoro M 1985 J. Physique Lett. 46217

Nishimori H and Stephen M J 1983 Phys. Rev. B 275644

Parisi G 1980a J. Phys. A: Math. Gen. 131101

—_ 1980b J. Phys. A: Math. Gen. 131887

- 1980c Phil. Mag. B 41677 1983 Phys. Rev. Lett. $\mathbf{5 0} 1946$

Proc. Heidelberg Conference 1983 ed. I L Van Hemmen and I Morgenstern (Berlin: Springer)

Sherrington D and Kirkpatrick S 1975 Phys. Rev. Lett. 351972

— 1978 Phys. Rev. B 174384 